\title{
Surgery for neuritis in leprosy: indications for and results of different types of procedures
}

\author{
RAYMOND BERNARDIN \& BERMAN THOMAS \\ Hopital Cardinal Léger, Institut Fame Pereo, 160 Avenue Poupelard, \\ Port-au-Prince, Haiti (W.I.)
}

Accepted for publication 26 November 1996

Summary From December 1988 to December 1992, 129 surgical procedures were performed on the peripheral nerves of 64 leprosy patients at the Hospital Cardinal Léger de l'Institut Fame Pereo for leprosy control in Haiti.

Sixty-four patients totalizing 129 nerves with sufficient clinical data form the basis of this study.

Based on the retrospective analysis of the operated cases, a new classification built on macroscopic findings of the involved nerves is presented. Five grades, according to the presenting aspects of these nerves, are set up as guides for different surgical procedures to be performed on the nerves: external decompression for the lesser grades I and II, intraneural neurolysis, interfascicular neurolysis for the higher grades III and IV, cleaning, and debridement for grade V. The final results are discussed. This new macroscopic grading done at surgery helps to minimize the aggressive procedures performed on nerve trunks, decrease the morbidity of surgical action on the nerve vascular structures, and consequently, preserves all possible sensory and motor functions of a nerve.

\section{Introduction}

All patients with leprosy present some form of nerve involvement at the skin level (hypoaesthesic or anesthesic lesion); it has even been described as some form of 'pure neuritic' leprosy without or before skin lesion, 'silent neurits' or 'quiet nerve paralysis' (QNP). However, most forms of nerve involvement are too of ten not recognized until the hands or feet are damaged.

Early detection and treatment of nerve trunk lesion in leprosy neuritis before permanent damage, loss of neural function, and subsequent disability, are the ultimate goals in dealing with neuritic leprosy. Once the nerve lesion is detected early, the physician, fieldworker, therapist, and all other personnel involved, hope that adequate and effective treatment is available in order to interrupt the downhill progression of the lesion, preserve the most valuable function of the nerve and, in so doing, protect the patient from a lifelong physical disability. 
Table 1. Distribution of nerves according to the proposed macroscopic grading

\begin{tabular}{lrrrrrr}
\hline GRADES & & II & III & IV & V & Total nerves \\
\hline Ulnar & 40 & 19 & 10 & 6 & 3 & 78 \\
Median & 22 & 11 & 4 & - & - & 37 \\
Common peroneal & 9 & 2 & 3 & - & - & 14 \\
\hline Total grades & 71 & 32 & 17 & 6 & 3 & 129 \\
\hline
\end{tabular}

\section{Materials and methods}

From December 1988 to December 1992, 129 surgical procedures were performed on nerve trunks in both upper and lower limbs of 64 patients at the Hospital Cardinal Léger of the 'Institute Fame Pereo'.

Thirty-six males and 28 females were registered. The age difference between male and female was not significant. The youngest patient was 8 years; the oldest 65 . Four patients were aged below 10 years; 28 were in their teens; 16 ranged between 20 and 30 years; seven, five and three were respectively within 40, 50 and 60 years. One patient was 65 .

From a total of 129 nerves which were operated on, the ulnar nerve accounted for 78 cases, the median nerve for 37 , the common peroneal nerve 14 . Seventeen patients did not present their nerve involvement at once. Thirteen patients required two operations at different times; four, three operations.

Bacteriological studies from these patients showed at $52(81 \cdot 2 \%)$ were paucibacillary and $12(18 \cdot 8 \%)$ multibacillary. In our series polyneuritis was more frequent in paucibacillary

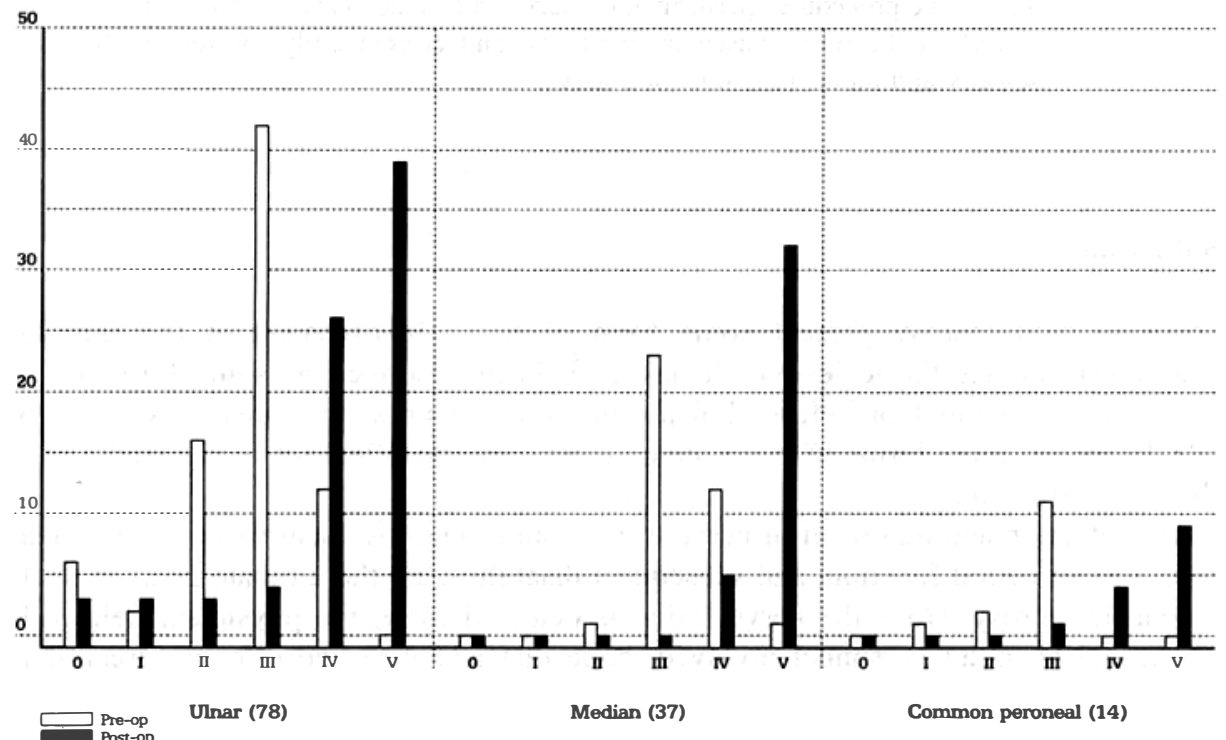

Figure 1. Pre- and postoperative motor assessment. 
patients: 46 out of 52; also mononeuritis seemed to be more typical of multibacillary patients: 7 out of 12 .

All these patients were evaluated for sites of tenderness along the nerve trunks, sweating of hands and feet, level of sensory as recorded on hands and feet charting, bone absorption of fingers and toes, status of finger joints motion, voluntary and manual testing of muscle strength in hands and feet, occupation, age and sex distribution. Prior to their diagnosis all the adult patients were involved in some profitable activities as farmers, housewives, street vendors and craftsmen. At the time of referral for orthopaedic evaluation the patients presented persistent and severe pain, severe paresthesia, sometimes muscle wasting and deformity. Detection of nerve involvement was based on the evaluation of voluntary muscle strength against resistance and sensory testing using Semmes-Weinsten monofilaments in order to assess the patient's touch-pressure recognition. A negative response to 4.56 (3.63 g) filament marking was considered as loss of protective sensation for the hand; and for the foot a negative response at $6.65(4.48)$. Assessment of motor function consisted in voluntary testing of selected muscles for each nerve: abductor digiti minimi, flexor carpi ulnaris and first dorsal interosseous muscles for the ulnar nerve; opponens pollicis and abductor pollicis brevis for the median nerve; tibialis anterior and extensor muscles of the toes for the common peroneal nerve. The pre-operative evaluation for motor and sensory functions of the involved nerves was done from a few days to 4 weeks prior to surgery. We found for the ulnar nerve, in preoperative assessment, no case with normal sensation (grade V), five cases with diminished light touch (grade IV), 42 with diminished protective sensation (Grade III), 27 with loss of protective sensation (grade II) and four with only deep pressure sensation (grade I). In motor testing there was no case of normal function (grade V), 12 of grade IV, 42 of grade III, 16 of grade II, 2 of grade I and 6 of grade 0.

For the median nerve, preoperatively, we found no case of normal sensation (grade V), 6 of grade IV, 27 of grade III, 4 of grade II and no case of grade I or 0 . Testing motor strength revealed one case of grade V, 12 of grade IV, 23 of III and one of II; there was no case of grade I or 0 .

For the common peroneal nerve, 11 cases were of sensory grade III, 2 of grade II and one of grade I; for the motor function there was one case of grade I, 2 cases of grade II, 11 of grade III.

Functional deficit of a nerve can be the result of increased external pressure on the nerve trunk which from hypertrophy cannot accommodate its larger size within the fibro-osseous channels such as the cubital tunnel at the elbow, carpal tunnel at the wrist, retro-fibular tunnel at the knee. This hypertrophy represents one of main characteristic changes in leprosy neuritis.

The main indications for surgery were:

failure of medical treatment in controlling the patient's symptoms after at least 4 weeks of $30-40 \mathrm{mg}$ of prednisone a day (17 patients);

complications from medical treatment such as gastritis, gastroduodenal ulceration, hypertension, psychotic disorders, toxicity (15 patients);

young age associated with severe symptoms (12 patients);

great severity of neural symptoms with excruciating pains (10 patients);

lack of compliance to the medical treatment ( 3 patients);

rapid deterioration of neural status ( 3 patients);

infectious process and immune deficiency (2 patients);

pregnancy (one patient); and

psychiatric disorder (one patient). 
Fifteen patients who obtained improvement of their symptoms from prednisone had to stop their medical treatment because of complications. These patients' charts mentioned only improvement of their neural symptoms without real assessment of the nerves sensory and motor functions.

At surgery the trunks of different nerves presented various macroscopic aspects which allowed us to realize different types of surgical procedures according to these aspects. In the first type of nerve lesion, hypertrophy of the trunk was obvious. However the nerve appeared normal in spite of a yellowish or brownish discoloration due to impregnation by medication, in particular clofazimine. The perinerve appeared shiny, glistening and transparent. At gentle palpation the nerve was supple with a regular consistency and free of any induration. For this type of lesion we performed an external decompression in which the constrictive fibrous ligament structures compressing the nerve were opened and excised, creating more space to accommodate the hypertrophied nerve trunk. In doing this ligamentotomy we made sure we relieved all the surrounding compressing fibrous bands or ligaments according to the anatomical specificity of the nerve involved; for example, in the case of the ulnar nerve, all three levels of possible compression, at the medial intermuscular septum, in the cubital tunnel and distal to the tunnel at the aponeurosis of the flexor carpi ulnaris muscle, should be explored and decompressed as necessary. During the course of this procedure all the vascular channels penetrating or leaving the epineurium should be left intact; the nerve should not be subjected to undue displacement from its bed, nor put under tension or traction through the use of rubber bands, gauze strips (umbilical tapes) or any other materials, for fear of tearing the fragile blood vessels (arterioles and veinules) on the posterior aspect of the nerve trunk. This is one of the main reasons the anterior transposition of the ulnar nerve and epicondilectomy are not done systematically.

From 78 ulnar nerves operated on in our series only 3 anterior transpositions (3.8\%) with epicondylectomy were performed.

In the second type of nerve trunk lesion, inflammation of the perinerve was evident; it lost its brilliance and shine; it looked pale and more or less opaque. There was an apparent state of vascular congestion. Some small areas of focal haemorrhage (probably as a result of trauma from rubbing and scratching) could also be noticed. Nevertheless the nerve was still supple, regular and without induration. At the opening of the nerve sheath, dissection of the bundles is quite easily performed because of little and rather loose interfascicular fibrosis formation and mild oedema of the axons. The primary purpose of this neurolysis is to decompress the inner structures of the nerve.

A more advanced macroscopic lesion showed thickening and irregularity of the epineurium along with its paleness and opacity. In addition they were short segments (1 to $5 \mathrm{~mm}$ ) of induration. At epineurotomy an important intraneural fibrosis and numerous interfascicular connecting fibrous bands could be found. Dissection of the fascicles was still possible. But the axonal fibres were the site of important oedema. Some of the fibres presented small areas of necrosis (softening); there were also marked vascular congestion. For this advanced lesion a more aggressive surgery was used. It consisted in longitudinal opening of the epineurium, dissecting off its edges in order to create sufficient room for the axonal bundles. In the course of dissecting the epineurium, the penetrating vessels should be left intact by making step-ladder, discontinuous types of incisions, or placing the axial incision away from the main vascular channels.

The fourth type of lesion is represented by long segments of induration along the nerve trunk, which made very difficult all attempts of intraneural dissection. The sheath is very 


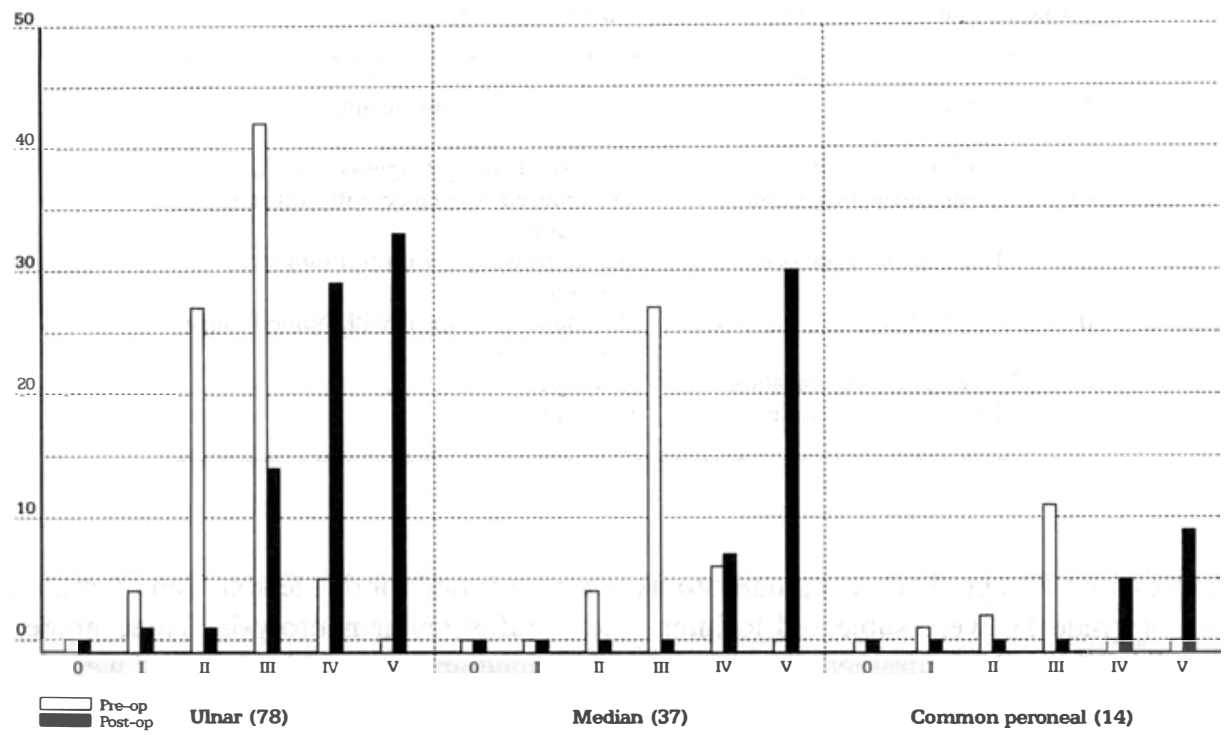

Figure 2. Pre-and postoperative sensory assessment.

thick, adherent, completely opaque and difficult to dissect off. In some areas the nerve trunk is transformed into a long hard cord by intense fibrosis. Softening of the axons and in some places, formation of small abscesses were seen as signs of advanced axonal necrosis. Surgery for this type of lesion was carried out inside the nerve fascicles, mainly the better preserved ones, following the same principles of vascular channels preservation. After opening the thick endoneurium, dissection reached some intact or partly intact axons which were freed from any persisting constriction.

The fifth type of lesion was represented by an abscess made of large areas of purulent collection along the nerve trunk. Sometimes the abscess had already burst through the skin (fistulization). There was also complete necrosis and liquefaction of the nerve around the site of the abscess. Surgery for this type V of lesion consisted essentially in cleaning the abscess which was washed out, drained and packed open. During a second procedure, 48-72 h or later if necessary, following daily dressing changes, the integuments could be repaired. At this time, fibrous ligaments causing compression on the nerve could be resected.

The macroscopic grading devised from operated cases serves as an indication of stage of lesions involving the nerve and its inner structures. The surgical procedures performed on various nerves were based on this grading for the following definite reasons:

To limit the surgery to strictly necessary action requiring decompression of the nerve and its axons.

To minimize, as much as possible, surgical trauma to the nerve structures, in particular to decrease the damage of the nerve vascular component to the minimum.

To reduce to the very minimum the postoperative morbidity to nerve functions.

To make the surgical act as simple as possible for the surgeon by providing him with some sound guidelines.

From the 78 ulnar nerves, at surgery 40 showed a grade I lesion and underwent a 
Table 2. Grading parameters for sensory and motor testing

\begin{tabular}{ll}
\hline Sensory testing & \multicolumn{1}{c}{ Motor testing } \\
\hline V- 2. 83: normal sensation & V: normal strength against resistance \\
IV- 3. 61: diminished light touch & $\begin{array}{l}\text { IV: reduced resistance with full range of } \\
\text { motion }\end{array}$ \\
$\begin{array}{ll}\text { III- } 4.31 \text { : diminished protective } & \text { III: no resistance with full range of } \\
\text { sensation } & \text { motion } \\
\text { II- } 4.56: \text { loss protective sensation } & \text { II: visible movement with limited range } \\
\text { of motion }\end{array}$ \\
$\begin{array}{ll}\text { I- 6. 65: deep pressure sensation } & \text { I: flicker } \\
\text { 0- complete loss of sensation } & \text { 0: paralysis }\end{array}$ \\
\hline
\end{tabular}

decompression; 19 a grade II, an epineurotomy was performed for this lesion. Ten of grade III and six of grade IV were subjected to inter- and intrafascicular neurolysis. Three grade V lesions were cleaned and drained. From a total of 37 median nerves, 22 of grade I were only decompressed; 11 of grade II and four of grade III were operated on following the same principles of neurolysis. The common peroneal nerve accounted for 14 nerves from which nine were of grade I lesion, two of grade II and three of grade III. Only the last two groups underwent epineurotomy and neurolysis; the first one only a decompression.

At follow-up (24-53 months) results for the ulnar nerve showed a good improvement of the nerve functions: 33 nerves (from nil) regained a normal sensory function (grade 5), 29 lost minor sensation (grade 4) and five pre-operatively. Fourteen nerves persisted with diminished

Table 3. Pre and post-operative sensory and motor assessment (follow-up 13 to 53 months-mean $32 \cdot 5)$

\begin{tabular}{|c|c|c|c|c|c|c|c|c|}
\hline \multicolumn{3}{|c|}{ Ulnar nerve (78) } & \multicolumn{3}{|c|}{ Median nerve (37) } & \multicolumn{3}{|c|}{$\begin{array}{l}\text { Common peroneal } \\
\text { nerve (14) }\end{array}$} \\
\hline Pre & Grade & Post & Pre & Grade & Post & Pre & Grade & Post \\
\hline \multicolumn{3}{|c|}{ Sensory } & \multicolumn{3}{|c|}{ Sensory } & \multicolumn{3}{|c|}{ Sensory } \\
\hline 0 & 0 & 0 & 0 & 0 & 0 & 0 & 0 & 0 \\
\hline 4 & I & 1 & 0 & I & 0 & 1 & I & 0 \\
\hline 27 & II & 1 & 4 & II & 0 & 2 & II & 0 \\
\hline 42 & III & 14 & 27 & III & 0 & 11 & III & 0 \\
\hline 5 & IV & 29 & 6 & IV & 7 & 0 & IV & 5 \\
\hline 0 & V & 33 & 0 & $\mathrm{~V}$ & 30 & 0 & V & 9 \\
\hline \multicolumn{3}{|c|}{ Motor } & \multicolumn{3}{|c|}{ Motor } & \multicolumn{3}{|c|}{ Motor } \\
\hline 6 & 0 & 3 & 0 & 0 & 0 & 0 & 0 & 0 \\
\hline 2 & I & 3 & 0 & I & 0 & 1 & I & 0 \\
\hline 16 & II & 3 & 1 & II & 0 & 2 & II & 0 \\
\hline 42 & III & 4 & 23 & III & 0 & 11 & III & 1 \\
\hline 12 & IV & 26 & 12 & IV & 5 & 0 & IV & 4 \\
\hline 0 & V & 39 & 1 & $\mathrm{~V}$ & 32 & 0 & V & 9 \\
\hline
\end{tabular}


protective sensation (grade 3 ) from 42 . Only two patients did not regain protective sensation against pressure from 27; and no case of complete loss of sensation from four preoperatively. For the motor function of the ulnar nerve-depending muscles, three cases remained paralysed (grade 0) and three with flicker motion (grade 1). Thirty-nine cases (from nil) regained normal strength on the tested muscles (grade V); 26 cases presented some reduced resistance (grade 4); and seven were found with very weak muscle strength (grade 3 and 2).

The median nerve, which totalled 37 cases, was found with preserved sensory function in all the cases: 30 presented a normal sensation (grade 5) and seven showed a diminished sensation to light touch (grade 4). Similarly strength of the tested muscles was normal in 32 cases (grade V); and five presented some mild weakness (grade 4) at voluntary muscles testing.

For the 14 common peroneal nerves, nine remained with normal sensation (grade V), and five with minor diminished sensation (grade 4). As far as motor function is concerned, nine cases presented at follow-up a normal resistance (grade 5), four minor loss of strength (grade 4 ) and one a grade III manifested by supple drop foot.

There were 7 minor (3.4\%) complications which were controlled by adequate measures:

Three 'stitch' abscesses which responded to topic antibiotics.

Two upper arm haematomas; one required drainage for 3 days, while the other one reabsorbed spontaneously.

Two wound dehiscences; one needed secondary sutures in the upper arm, while the other one was controlled with taping (steri-strips). There was also a case of an ulnar abscess at the Guyon's canal which required, for control, a second operation 3 weeks after the first surgical intervention.

\section{Discussion and conclusion}

Setting up a clinical classification in leprosy neuritis that can serve as a guide for the precise surgical procedure to be performed, has been a challenge throughout the years. Surgery is used as a helpful tool in the armarium of means for relieving and controlling this microbacterial nerve involvement. The classification by Srinivasan of the affected nerves according to stages of 'involvement', 'damage', and 'destruction' is one that describes the severity of the peripheral nerve lesion. However, this classification does not help the surgeon much because the findings by palpation through the skin of an hypertrophied, thick, tender nerve does not indicate the actual condition of the nerve lesion. This is complicated by the fact that damage to a nerve can be established at once from the onset of neuritis at the time the patient is seen. Furthermore, it is not always quite predictable to state that a particular nerve has absolutely no chance to partially recover some of its function.

The ultimate goal is to diagnose the neuritic condition before irreversible, functional deficit occurs. For in the type of entrapment neuropathy found in leprosy, the anoxal damage is related to both the immunologic phenomena responsible for all the inflammatory modifications and pathological changes in the nerve and also the hemodynamic alteration in the vascular system of the nerve in term of compression of the arteriolar walls, with direct consequences of anoxal ischemia. The definite treatment should be applied before any irreversible changes or destruction reach the axons, and should achieve the following two goals:

decrease hypertension around and within the nerve; and prevent ischemia. 
Besides the specific antibacterial therapy, the immunosuppressive effect of certain drugs such as corticosteroid agents, the sedative and analgesic properties of thalidomid, and antiinflammatory ones of clofazimine are used to relieve pain and control hypertrophic neuritis. The various success of these drugs is indisputable. However, the following limitations do exist:

They must be used from the very beginning of the neuritis before ischimia takes place. Their use can be for prolonged periods of time and at very high doses (such as prednisolone for up to 6 months). At such doses (starting dose 40-60 mg per day) and side-effects (mental, endocrine, gastroduodenal) may very much limit their use.

Those drugs are usually used in association with rest and immobilization of the involves limb or limbs, sometimes for long periods of time (6-8 weeks or more). This immobilization may represent quite a handicap for the patient with multiple nerve involvement.

Their use does not guarantee success as far as the control of severe symptoms is concerned, nor prevent the downfall progression of the nerve lesion. Surgical decompression and neurolysis, properly and completely done (in particular, in the case of the ulnar nerve), have an immediate effect on relieving pain, decompressing the nerve at once, and relieving the compression of the axons and the vascular structures of the nerve. In doing so there is no need for immobilization; and both techniques serve as a sure, definite, and immediate way to prevent ischemia of the nerve and should be carried out:

Immediately, in cases of severe, acute, hyperalgic neuritis; mainly if the antiinflammatory drugs cannot be used because of other medical conditions (such as infections, pregnancy, gastroduodenal ulcers, mental disorders, hypertension, diabetes) or if some of the drugs (Thalidomid in our case) are not available.

Surgery can be delayed in cases where these drugs and immobilization are used and the patient shows overt clinical improvement of his/her symptoms for 3 weeks at the most. If in this delay, there is no sign of improvement or even worsening in the nerve condition, in spite of the treatment, one should immediately consider surgery.

For such neuritis of less than 1-year duration with apparent sensory loss, muscle weakness, and paralysis (macroscopic lesions of grade III and IV) surgery can still be of value, allowing that intact axons, not yet touched by necrosis, especially the 'small, slower conducting fibres' which are more resistant to elevated pressure than the 'large, fast conducting fibres" can be saved through internal decompression.

Even for long-term lesions, in particular for cases of insensitive feet with plantar ulcer, after healing the ulcer by successive, pressure-control casting or other means, the decompression surgery of the posterior tibial artery can offer some hope in preventing the recurrence of these ulcers by way of increasing vascularization of the plantar tissues and improve the proprioceptive function and neural feedback of those tissues.

\section{References}

${ }^{1}$ Hargens AR, Romine JS, Sipe JC, Evans KL, Mubarak SJ, Akeson WH. Peripheral nerve-conduction block by high muscle-compartment pressure. J Bone Joint Surg 61-A:2; March 1979, 192-200.

2 Srinivasan H. Prevention of disabilities in patient with leprosy. A practical guide WHO Geneva 1993. 